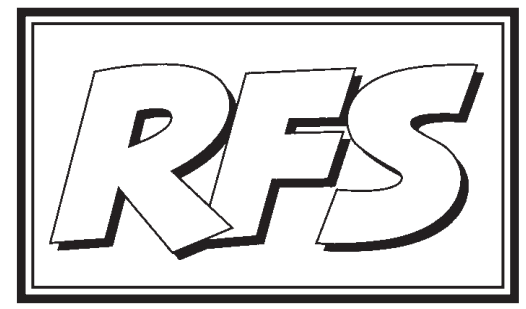

Revista de Fomento Social, 61 (2006), 455-464

\title{
El Foro de alto nivel sobre la Agenda Social de la Integración Centroamericana y las relaciones UE / Centroamérica. Una crónica
}

\section{Introducción}

Organizado por el Centro Internacional para el Desarrollo Humano ${ }^{2}$ y el Círculo de Copán ${ }^{3}$, el Foro se desarrolló el 9 y 10 de Octubre en San José, Costa Rica.

1 Economista y consultor. Funcionario y Director de planificación del Banco Centroamericano de Integración Económica (BCIE) hasta el año 2004. Colaborador de la Fundación ETEA para el Desarrollo y la Cooperación.

2 El Centro Internacional para el Desarrollo Humano (CIDH) es una organización de investigación no lucrativa, multidisciplinaria, educativa y de investigación aplicada. Ubicada en San José, Costa Rica.

3 El Círculo de Copán es una red generadora de pensamiento y orientaciones estratégicas 
Este evento forma parte de las actividades realizadas en el marco de la presidencia pro tempore del Sistema de Integración Centroamericana del gobierno de Costa Rica (segundo semestre de 2006).

Las ponencias estuvieron a cargo de Óscar Arias (Presidente de Costa Rica), Eneko Landaburu (Director General de Relaciones Exteriores de la Unión Europea), Aníbal Quiñónez (Secretario del Sistema de Integración Centroamericana), Rubén Zamora (Círculo de Copán, El Salvador), Bruno Stagno (Canciller de Costa Rica), Haroldo Rodas (Secretario del Sistema de la Integración Económica Centroamericana), Federico Carrillo (Vicepresidente del Banco Centroamericano de Integración Económica) y Miguel Hakim Simón (ex Comisionado del Plan Puebla Panamá) por México, entre otros.

A continuación exponemos una síntesis de las intervenciones más relevantes de este importante foro ${ }^{4}$.

\section{Oscar Arias: libre comercio y tareas pendientes en Centroamérica}

La inauguración estuvo a cargo del Presidente Oscar Arias Sánchez. Al plantear su visión del libre comercio y la región, expuso las siguientes ideas.

El principal argumento para justificar el libre comercio es su contribución a reducir la pobreza, como lo constata en el continente el ejemplo de Chile. $\mathrm{Al}$ presidente Arias le maravilla cómo puede haber gente que se opone y hasta lo califica de perverso.

Ahora bien, pese a sus efectos benéficos, el libre comercio no basta. Hay que tomar medidas que son responsabilidad de los propios países, en complemento a la liberalización, a fin de aprovechar los beneficios. Estas obligaciones serían, entre otras:

- Aumentar la inversión social e invertir con orden y equidad. La prioridad de la inversión pública debe ser la educación, lo que en otros países implica reducir el gasto militar. No es ético que se engorde el armamentismo con pueblos desnutridos. Este es precisamente el Consenso de Costa Rica: que los países desarrollados aumenten su ayuda oficial al desarrollo (AOD) si nosotros gastamos con orden y equidad. Ojalá

sobre la integración regional centroamericana, con participación de políticos, expertos, académicos, dirigentes de organizaciones de la sociedad civil, de los diferentes países.

4 Los subtítulos son nuestros. 
ahora en "días claros" se nos ayude y no sólo cuando vivimos épocas de confrontación.

- Elevar la competitividad y democracia de los países, integrando procesos industriales, creando encadenamientos productivos, invirtiendo en infraestructura y desarrollando habilidades comerciales en las empresas.

- Implementar políticas públicas que dirijan los recursos presupuestarios a prioridades que eleven la productividad, preserven la biodiversidad y mejoren la equidad, así como al establecimiento de un Estado de Derecho que es condición sine qua non de la competitividad. Esto exige una reforma tributaria en cada nación para poder financiar las inversiones, reduciendo el gasto militar.

Por otro lado, las prácticas democráticas y la independencia de poderes asegurarán un sistema de pesos y contrapesos necesario para aprovechar la apertura, atraer inversiones y garantizar la gobernabilidad. Nada lastima más al Estado democrático que la corrupción, porque deslegitima a las instituciones democráticas.

El paso de la integración centroamericana se ha tornado lento e incierto. Cuando en 1987 se alcanzó un mecanismo para lograr la paz firme y duradera, y luego se adoptaron consensos importantes hasta 1994, se pensaba que se abría la esperanza de un nuevo proceso integrador en la región; pero, por el contrario, las asimetrías han aumentado y los países de la región se dividen en dos mundos en términos del Índice de Desarrollo Humano (IDH).

En todo caso, hay que trabajar para potenciar la integración centroamericana. Es imperativo aumentar la integración física; acercarnos al arancel externo; adoptar procedimientos aduaneros uniformes e implementar mecanismos creíbles para la solución de controversias. Construir la Unión Aduanera va a suponer pasar primero por negociaciones complejas, especialmente para solventar la lista de productos sensibles. Igualmente hay que consolidar el espacio político regional. No hay nada escrito en las estrellas, ni edictos, ni razones para profetizar que se ha garantizado ya la libertad y progreso de que hoy goza Centroamérica.

Es imperativo revisar el sistema de integración desde su base, a fin de enrumbarnos a la integración con la economía internacional. El PARLACEN, por ejemplo, es oneroso por su conformación y funciones, además de estar desprovisto de una visión clara y una agenda consecuente con los retos. 
Hay que reconocer las diferencias entre los países centroamericanos. Al Acuerdo de Asociación, la UE viene con una sola voz, no así los centroamericanos. Costa Rica no acepta un negociador único, en su lugar propone el mecanismo que con buen suceso se empleó en la negociación del CAFTA, donde la coordinación fue rotativa.

Debemos tener cuidado de no sucumbir a la tentación de pretender fortalecer a las instituciones regionales con este enfoque de negociación. Sería ceguera pretende asumir una homologación entre las instituciones de la UE y las centroamericanas, en razón de similitudes superficiales. Centroamérica es un cementerio de oportunidades cruelmente mutiladas...

\section{Eneko Landaburu: perspectivas de la integración centroamericana desde la óptica europea}

El encargado de las relaciones externas de la UE se planteó la interrogante de si seremos capaces de pasar a compromisos superiores para que dos realidades regionales sean motor de cambio en el mundo, en consonancia con la decisión tomada en Viena en mayo de 2006 para iniciar negociaciones hacia un Acuerdo de Asociación.

Al reconocer los éxitos de Costa Rica en materia de paz, democracia y desarrollo social, expresó que se comprueba que la inversión social y la atracción de inversión extranjera directa (IED) no son contradictorias. Sin embargo, a Costa Rica le urge elevar su inversión social por el gran deterioro de los indicadores, según constata el Informe del Estado de la Región. Sin cohesión social no podrá haber progresos en el crecimiento, la integración y la democracia. "En Bruselas estamos preocupados porque en América Latina, pese a los avances en la democracia y la estabilidad macroeconómica, las disparidades van en aumento".

Con el emprendimiento del Acuerdo, los principios de base de las relaciones entre la UE y Centroamérica no han cambiado. El marco sigue siendo triple: diálogo político, cooperación y comercio. Eso sí, sujeto a una negociación que tiene como base el regionalismo abierto y con flexibilidad de adaptación para tomar en cuenta las diferencias. Desde que Delors propuso para Europa el mercado interior como motor, sujeto a que los grupos importantes de las sociedad civil abrazaran un proyecto político, Europa es un continente de paz, próspero, democrático y solidario. 
Las relaciones UE / CA han cosechado éxitos en los últimos 20 años:

- El Acuerdo de San José fue el primer diálogo político de la UE con una región en su conjunto.

- La cooperación del período 2002-2006 ha representado desembolsos por 600 millones de Euros para 35 millones de habitantes, con el propósito de financiar proyectos de desarrollo y para atender desastres y conflictos civiles, con recursos provenientes del bolsillo de la gente de 25 países, que llegarán a 27 a partir del $1^{\circ}$ de enero de 2007.

- En las relaciones económicas, los países han podido aumentar los flujos de inversión y comerciales, con un $90 \%$ de las exportaciones a la UE bajo el Sistema Generalizado de Preferencias. El Acuerdo de Asociación que se propone hace que las perspectivas económicas sean aún más alentadoras.

Las características de este Acuerdo de Asociación UE-Centroamérica son:

- Es un acuerdo global, puesto que incluye las tres áreas de la relación, dentro de un marco contractual único. Es más que un simple Tratado de Libre Comercio.

- Es un acuerdo que se negocia entre dos bloques. Las expresiones del Presidente Arias generan discrepancias con lo que la UE piensa en materia de negociador único. Esta posición se deriva del Acuerdo de Cooperación de Roma de 2003. Si queremos tener éxito en la Cumbre de Lima de 2008, debemos hacerlo. No hay que temer a una pérdida de soberanía. El hecho es organizarse de tal forma que haya capacidad de seguimiento y máximo impacto del Acuerdo.

El diálogo político se orienta ahora a defender conjuntamente en foros internacionales los intereses comunes, elevar la capacidad de iniciativa y concentrarnos mejor en líneas estratégicas más claras, en torno a temas como la cohesión, los derechos humanos, los derechos laborales, la democracia, la violencia y el crimen organizado.

La UE cuenta con recursos para seguir apoyando a Centroamérica, pese a las dificultades previas a la concreción del presupuesto de cooperación para 2007-2013.

En materia de libre comercio, sin duda tendremos dificultades ya que un buen acuerdo es aquel que toma en cuenta los intereses de cada parte. No habrá tabúes y todo estará sobre la mesa. 
Sobre la integración regional, hay que recordar que no es un fin en sí misma. La UE la entiende como un método, no un objetivo, para alcanzar soluciones en beneficio de la ciudadanía. Es un proceso de largo plazo para alcanzar paz y prosperidad, pero Europa, pese a sus logros, no pretende dar lecciones a nadie.

El papel de la cohesión social y territorial, lo que equivale a hablar de solidaridad, es central en la estrategia de la UE para con Centroamérica. Todos debemos añadir la cohesión social como objetivo estratégico, con la misma determinación que lo hemos hecho con los derechos humanos y el Estado de Derecho. Es un valor fundamental de la UE: mercado más políticas de contrapeso y producción de cambios en la actitud social hacia la exclusión.

\section{Aníbal Quiñónez: los desafíos sociales de la integración centroamericana}

El secretario del SICA planteó que la integración centroamericana es una creación jurídica políticamente sustentada, en el marco de políticas estructurales en campos tan diversos como la pobreza, el desempleo, la corrupción, educación y salud, seguridad ciudadana y crimen organizado.

Propuso 9 desafíos sociales para la región, abogando por más cooperación internacional por la naturaleza compleja de los fenómenos:

- Luchar contra la pobreza extrema. Existe una población mayoritariamente joven que enfrenta la opción de emigrar o delinquir.

- Promover una acción multilateral para atender los rezagos sociales con una estrategia multisectorial, dado que los fenómenos están interrelacionados. Se requiere de la acción concertada de naciones con mayores recursos monetarios.

- Construir pilares democráticos fuertes (participación, Estado de Derecho).

- Luchar contra la vulnerabilidad (especialmente en los temas relativos a la vivienda y en el mundo rural).

- Crear oportunidades para la niñez y juventud, reorientando la inversión social y evitando así su integración a las maras.

- Vencer la fragilidad de los sistemas de salud y seguridad social. 
- Integrar la institucionalidad regional, fomentando la producción de bienes públicos regionales.

- Garantizar el acceso universal y de bajo costo al agua potable.

- Fomentar la justicia y equidad de género.

Los proyectos regionales no son sustitutos de la responsabilidad de los gobiernos en la provisión de bienes y servicios.

Ante la pregunta de si es posible enfrentar con éxito esos nueve desafíos con un esquema gubernamental de integración, el Secretario del SICA respondió, en un principio, que esa pregunta mejor sería pasársela a los Estados, para luego afirmar que el esfuerzo para hacer frente a los retos es gradual y que depende de la voluntad y decisión política de los países. Se pueden superar las brechas pero no es fácil.

Existe un enorme desorden en materia de arreglo institucional en el SICA. Existen 31 instituciones y 13 secretarías y un sinnúmero de decisiones presidenciales y temas de agenda. Se celebran muchas cumbres presidenciales con poco impacto, pero se hacen esfuerzos para aglutinar, depurar y priorizar los mandatos todavía válidos. Este año en Panamá se produjo por primera vez un Plan Operativo conjunto para toda la institucionalidad.

\section{Miguel Hakim Simón: el acuerdo con la UE y los retos de la región}

En la medida que avance el proceso de Doha, así avanzará con mayores probabilidades de éxito el Acuerdo de Asociación UE / CA. Hay que buscar asesoría en negociación con México, Chile, España y Portugal.

Asimismo, hay qué definir de qué estamos hablando al manejar el concepto de cohesión social, ya que para los europeos significa "cómo el desarrollo económico tira del desarrollo social", mientras que para los iberoamericanos significa "cómo combatir la pobreza y reducir la desigualdad". ¿Es un tema transversal o un objetivo?

Atraer Inversión Extranjera Directa es un imperativo, por los bajos niveles de ahorro regional. Pero no hay que perder de vista que las dos principales fuentes de financiamiento de Centroamérica son el comercio (\$90 mil millones) y las remesas ( $\$ 6$ mil millones), montos superiores a la misma IED $(\$ 2,8$ mil millones) y la AOD (\$2,5 mil millones). 
Construir la cohesión social, el contrato social, implica hacer la reforma fiscal, dado el bajo promedio de la carga tributaria que apenas llega al 13\%, variando desde un 9,6\% en Guatemala a un 17,2\% en Honduras. Es un objetivo difícil de lograr por el mayor tamaño del sector informal que el formal en la economía.

Surgirán asimetrías, pero el pilar de la cooperación ayudará a suavizarlas. Aunque todavía el nivel medio del IDH en Centroamérica oscila entre los valores 0,5 y 0,8 , en el tiempo ningún país ha sufrido retrocesos.

En materia comercial, somos segunda prioridad recíprocamente. Mientras para la UE el comercio intrarregional representa el 73\% de su comercio, para Centroamérica sus mayores socios son los Estados Unidos (38\%) y la misma región (17\%).

En síntesis, los beneficios del Acuerdo de Asociación con la UE son:

- Certeza jurídica con respecto al SGP.

- Mayor diversificación del comercio.

- Posiciones comunes en la ONU.

- Mayor flujo de IED a Centroamérica en turismo, energía, agroindustria, telecomunicaciones, construcción y ciertas ramas textiles.

\section{Federico Carrillo: el Banco Centroamericano de Integración Económica}

El propósito del BCIE es ser un banco de desarrollo para el sector privado con soluciones financieras sostenibles.

Su rol lo conciben por ello en el marco de dos ejes: impacto en las ordenadas y solidez en las abscisas. Su posición la ubican en el primer cuadrante (mayor impacto y solidez). Descartan el segundo por no ser posible (tener impacto sin solidez); el tercer cuadrante implicaría la liquidación (sin impacto ni solidez), y no les interesa el cuarto cuadrante porque significaría transformarse en banco comercial (sólido pero con escaso impacto).

Su concepción del desarrollo es multidimensional: fomentar el crecimiento del PIB y generación de empleo formal; mejorar la distribución del ingreso; aumentar el acervo de infraestructura; elevar la competitividad y fortalecer las instituciones públicas.

Su concepción estratégica es la de ser un catalizador (en el sentido de 
provocar combustión) para atraer inversión con alto impacto en el desarrollo. Esto se realizaría vía productos y servicios en banca de inversión (a fin de no endeudar a países se utilizarán esquemas como la titularización de flujos, concesiones y operaciones con Administradoras de Fondos de Pensiones) así como mediante servicios financieros (banca tradicional) y preinversión.

Llevar adelante su visión requiere atacar obstáculos, tales como la baja capacidad de endeudamiento y gestión de proyectos del sector público; la poca profundidad financiera e infraestructura del sector financiero y la capacidad financiera de las empresas.

Los sectores de alta prioridad son las MIPYMES, vivienda, la tecnología, la infraestructura, y energía y ambiente.

Expresó su opinión de que el desarrollo no se logra con Fondos de Cohesión como el aprobado en Belice ${ }^{5}$.

El BCIE recién comienza a desarrollar productos para bancarizar y reducir márgenes en el envío de remesas con un nuevo producto que está en proceso de aprobación, llamado Credencial Remesas. Asimismo, explicó que el Banco no proporciona financiamiento en educación o salud porque no es el Ministerio de esas carteras nacionales. No hay acción directa ya que los países tienen la organización para atender esas demandas. Lo que hace el BCIE es mejorar cada día para captar fondos en mejores términos y canalizar esos recursos en las mejores condiciones para los países.

Ante la pregunta de qué le hace falta al BCIE si concentra lo mejor de recursos humanos y financieros en Centroamérica, luego de un largo momento para responder, dijo que lo que necesitaba era más transformación interna, cambiar la estructura de gobierno que limitaba el cambio por ser los países los dueños, elevar el dinamismo operacional, modificar las actitudes del personal y mejorar la capacidad de ajuste de la institución.

5 En la Cumbre presidencial centroamericana de diciembre de 2003 en Belice, los presidentes acordaron "instruir al BCIE y a la SIECA a presentar, en el mismo plazo, un mecanismo de financiamiento para la conformación de un fondo de cohesión social para mitigar posibles impactos derivados de la Unión Aduanera y potenciar sus beneficios". 


\section{Conclusión}

El Foro resultó un espacio privilegiado, tanto por su organización, ponentes y audiencia, como por abordar lo social, dimensión relegada en las primeras cuatro décadas desde el Tratado General de Integración Económica. La discusión de temas de alcance regional, desde la perspectiva social, añade una esperanza a los que valoramos a la integración como un eje central para abordar el futuro de Centroamérica con mayores probabilidades de alcanzar el elusivo éxito de forma simultánea en lo económico, político y social.

Persisten disonancias que habrá que abordar con visión de estadistas; subsisten visiones parciales de los grupos de interés más determinantes en cuanto al papel e impacto del proceso de integración, y el tema de la cohesión social sigue siendo un aspecto no del todo asimilado, pero se vuelve a relanzar la discusión... y el tiempo sigue corriendo de cara a la Cumbre de Lima. 